\title{
Predicting the Corrosion Initiation Time in Reinforced Concrete Bridges Using Stochastic Simulation Methods
}

\author{
Abdelrahman Abdallah* \\ Colorado State University, Fort Collins, USA
}

*Corresponding author: Abdelrahman Abdallah, Research Assistant, Department of Civil and Environmental Engineering, Colorado State University, Fort Collins, CO 80523, USA.

\author{
Received Date: July 31, 2020 \\ Published Date: August 20, 2020
}

\begin{abstract}
Reinforced Concrete Bridges are considered one of the main components of the transportation infrastructure. These bridges require regular inspection and maintenance in order to be able to carry the applied loads. Predicting the behavior of concrete bridges and the time their condition state changes is crucial for providing better bridge management. Corrosion is considered one of the main reasons behind bridge deterioration. In this paper the time of corrosion initiation and the probability of exceeding a critical chloride level in the concrete substrate is evaluated, using Stochastic simulation methods. A probabilistic analysis was done considering the uncertainty associated with a corrosion initiation prediction model and associated parameters. The analysis process was divided into three main stages. In stage 1, Monte Carlo Simulation (MCS), Importance Sampling (IS) and Taylor Expansion Series were used to predict the corrosion initiation time. In stage 2 the probability to exceed the chloride threshold level was considered as the probability of failure and was estimated at different time periods using Monte Carlo Simulation (MCS), Importance Sampling (IS) and First Order Reliability Method (FORM). Finally, in stage 3 model parameters were updated using Bayesian updating. Inspection data measurements were assumed for the updating process.
\end{abstract}

Keywords: Reinforced concrete bridges, Chloride induced corrosion, Monte carlo simulation (MCS), First order reliability method (FORM), Importance sampling (IS), Taylor expansion series, Bayesian updating

\section{Introduction}

Reinforced concrete bridges are a critical component within the transportation infrastructure. These bridges are facing continuous deterioration due to increasing traffic loads and aggressive environmental conditions [1]. Regular inspections and maintenance actions are required to keep these bridges capable of carrying out its intended function at a satisfactory level. The main challenge in making accurate maintenance and inspections decisions is due to funding constraints on bridge managers. A proactive approach to bridge management is likely to provide optimal economical and effective management decisions [2]. The quality of these decisions depends on successful prediction of civil infrastructure's future condition state. Many researches has proposed different types of deterioration models to predict the condition of concrete bridges at different stages during its life span $[3,4]$ Stochastic models, have been used in predicting the deterioration process of reinforced concrete bridges. One of the main advantages of stochastic models is their ability to capture the uncertainty when predicting the condition of a reinforced concrete bridge at different time periods [5]. Chloride-induced corrosion of steel in reinforced concrete structures is one of the major causes of reinforced concrete deterioration over time. In the winter season deicing salts are spread on roads to keep them safe and to avoid traffic collision. However deicing salts are considered one of the main sources of chloride accumulation on the bridge deck. Chlorides from de-icing salts or marine breeze penetrate through the concrete cover affecting the protective oxide layer formed around the reinforcements [6]. As the chloride content reaches the threshold level at the rebar level, the protective film surrounding the rebar fails and corrosion initiates. Due to the 
volume increase, tensile stresses are induced on the concrete substrate around the rebar causing cracking or spalling of the concrete cover [7].This paper will focus on the chloride accumulation phase which starts when chloride accumulate on the concrete surface until corrosion initiation time.

\section{Research Objectives}

In the present paper the chloride accumulation deterioration model proposed by [8] will be used to predict the time at which corrosion will initiate in a reinforced concrete bridge which is assumed to be exposed to deicing salts. Probabilistic information about uncertainties related to the environment and physical properties of the materials have been estimated from a wide review of previous experimental or statistical studies. The probability of failure at different time periods will be calculated using a proposed threshold value for chloride content. To perform the above predictions, different stochastic methods will be used, Direct Monte Carlo Simulation (MCS), First Order Reliability Method (FORM), Taylor Approximation and Importance Sampling (IS). Finally Bayesian updating will be done on the model parameters using assumed on-site inspection results.

\section{Deterioration Model}

In most chloride induced deterioration models, degradation of the concrete deck is not considered until corrosion has initiated at the rebar level [4]. However, this approach undermines the benefits of choosing an optimum inspection time and taking any proactive maintenance measures. Rafiq et al. [9] suggested considering the chloride accumulation stage as part of the bridge deterioration, this can be achieved by defining the propensity to corrosion as a performance measure for a concrete bridge [9]. Previous research in marine and bridge structures, has shown that chloride accumulation profiles in concrete can be approximately modeled by integrating Fick's second law [8]. As shown by equation (1)

$$
C(x, t)=C_{0}\left[1-\operatorname{erf}\left(\frac{x}{2 \sqrt{D t}}\right)\right](1)
$$

where $\mathrm{C}(\mathrm{x}, \mathrm{t})$ is the chloride content at a distance $\mathrm{x}$ from the surface , Co $\left(\mathrm{kg} / \mathrm{m}^{3}\right)$ represents the chloride concentration at the deck surface, $\mathrm{D}\left(\mathrm{m}^{2} /\right.$ year) represents the diffusion rate, $\mathrm{x}(\mathrm{m})$ represents the depth of interest below surface(i.e. the cover depth for concrete decks), and t represents the time period to be analyzed[8]. This formula can be re-arranged to estimate the corrosion initiation time, and the $\mathrm{C}(\mathrm{x}, \mathrm{t})$ can be replaced by the critical threshold value $\mathrm{C}_{\mathrm{th}}\left(\mathrm{kg} / \mathrm{m}^{3}\right)$, which indicates the critical chloride concentration level for corrosion to initiate[9,10].

$$
t_{\mathrm{o}}=\frac{1}{4 D}\left(\frac{x}{\operatorname{erfc}^{-1}\left(\frac{C_{t h}}{C o}\right)}\right)^{2}
$$

$\left(t_{0}\right)$ is the time at which chloride level will reach the threshold value and corrosion will start.
The values of the parameters used in the models are not known with certainty and can vary for different bridge structures and locations; thus each parameter was considered as a random variable. Distribution types and the moments of the random variables are indicated in Table (1). A model error factor was used to account for the uncertainty associated with the model (Emod), also another error factor was used to account for the uncertainty used for the prediction of the diffusion coefficient (D), which mainly depends on the content of the concrete mix [4,9] (Tables 1$)$.

The values for the following parameters were provided from a wide range of literature reviewed $[2,4,9,11]$.

\section{Methodology}

The methodology of this paper consists of three main stages, prediction of time at which corrosion will initiate, evaluation of the probability of failure at different time periods and finally updating model parameters using Bayesian updating. In stage 1 prediction of corrosion initiation time will be done by evaluating equation (2) using three stochastic methods; Monte Carlo simulation (MCS), Importance sampling (IS) and Taylor approximation. The general stochastic integral used in the calculations is shown in equation (3):

$$
H \triangleq E_{f}[g(\xi)]=\int_{E} g(\xi) f(\xi) d \xi=\int_{\Xi} k(\xi) d \xi
$$

Where $f(\xi)$ is the PDF of the estimated parameter and $g(\xi)$ is the scalar function of the random variable. The predicted value and the coefficient of variation (CV) of the three methods will be compared. The uncertainty associated with each method in this paper will be referenced using the CV. In stage 2 the probability of failure will be calculated using equation (1) and comparing it with the threshold chloride level $\mathrm{C}_{\text {th }}$. In stage 2 the probability of failure will be calculated using Monte Carlo simulation, Importance sampling, and First Order Reliability Method (FORM). The general indicator function used to describe the probability of failure is shown in equation (4):

$$
P_{F}=E_{f}\left[I_{F}(\xi)\right]=\int_{\Xi} I_{F}(\xi) f(\xi) d \xi
$$

A comparison between the results of the different methods will be provided [12]. In the final stage inspection results will be used to update the parameters of the integral in equation (1). The inspection results are assumed to be associated with two Non-destructive tests, Cover meter and Chloride Ion Penetration method. The cover meter is used to measure the depth of the concrete cover. The chloride ion penetration method measures the chloride content in the concrete which is the same measurement equation (1) predicts $[13,14]$.

\section{Direct Monte Carlo Simulation}

Monte Carlo simulation is mainly based on the Central Limit Theorem. After generating samples for each random variable, simulations are done to evaluate the integral( $(\xi)$ which in our case is the corrosion initiation time in stage 1 or the chloride content in the 
concrete deck at a certain time period in stage 2 . The average value H_K of the integral result is calculated by dividing the summation of the integral samples by the number of simulations $\mathrm{K}$ as shown in equation (5)

$$
\hat{H}_{M C S, K}=\hat{H}_{K}=\frac{1}{K} \sum_{i=i}^{K} g\left(\xi_{i}\right)
$$

The coefficient of variance (CV) associated with MCS is calculated using equation (6)

$\mathrm{Cv}=$

$$
\frac{1}{\sqrt{K}} \frac{\sqrt{\operatorname{variance}_{\left\{\xi_{k}, k=1, \ldots, K\right\}}(g(\xi))}}{\operatorname{mean}_{\left\{\xi_{k}, k=1, \ldots, K\right\}}(g(\xi))}
$$

The CV can be used to evaluate the accuracy of the estimator and the effect of the number of simulations on the prediction accuracy [12]. B When using MCS to calculate the probability of failure the only difference is that the evaluated average of the chloride content is compared to the threshold value. An indicator function is used to count the number failures associated with each simulation and using it to calculate the probability of failure with an associated CV [12].

\section{Importance Sampling}

Importance sampling is used to generate samples for the random variables that are more likely to reach the optimum integral prediction. It's expected that the $\mathrm{CV}$ associated with an importance sampling estimator to be lower than the CV associated with MCS. When using importance sampling, it's assumed that equation (3) can be written as (7):

$$
\left.H=E_{f}[g(\xi)]=\int g(\xi) \frac{f(\xi)}{q(\xi)} q(\xi) d \xi=E_{q}[g(\xi)) \frac{f(\xi)}{q(\xi)}\right]
$$

In Importance sampling an optimal sampling density $\mathrm{q}^{\wedge *}(\xi)$ is assumed which is realted to the main integrand [12]. The optimal sampling density equation is represnted by the following equation (8):

$$
q^{*}(\xi)=\frac{|g(\xi)| f(\xi)}{\int|g(\xi)| f(\xi) d \xi} \propto|g(\xi)| f(\xi)
$$

The CV of the importance sampling can be caluclated using equation (9):

$$
\delta_{I S, K} \approx \frac{1}{\sqrt{K}} \frac{\sqrt{\text { variance }_{\{\xi, k, \ldots, \ldots, K\}}\left(\frac{g(\xi) f(\xi)}{q(\xi)}\right)}}{\operatorname{mean}_{\{\xi \xi, k=1, \ldots\}}\left(\frac{g(\xi) f(\xi)}{q(\xi)}\right)}
$$

In calcaulting the probality of failure in stage 2 the mean and variance of the failure integral is used in sampling the failure events [12].

\section{Taylor Series Expansion}

Taylor series expansion or Taylor Approximation is based on information provided regarding the integrals. It changes equation (3) to this format (10):

$$
H=\int_{z} g(\xi) f(\xi) d \xi=\int k(\xi) d \xi=\iint_{z} e^{\ln (k(\xi))} d \xi
$$

Where the integrand is taken to the exponential level or optimized. The average value of our integral is approximately found by identifying the design point and evaluating the Hessian of the integrand and its log function; equation (11) shows the integral estimated:

$$
H \approx \frac{(2 \pi)^{n / 2} k\left(\xi^{*}\right)}{\sqrt{\left|\operatorname{det} \mathbf{H}_{\ln k}\left(\xi^{*}\right)\right|}} \approx I
$$

wherek( $\left(\xi^{\wedge *}\right)$ is the integrand evaluated at point $\xi^{\wedge *}, \mathrm{n}$ is the number of parameters, and $\left[\mathrm{H} \rrbracket \_\ln\right.$ k $\left(\xi^{\wedge *}\right)$ is the Hessian of the log of the integrand evaluated at $\xi^{\wedge *}$. It should be noted in this paper numerical differentiation was done in order to perform the optimization and get the Hessian values which is different to what was done in importance sampling [12]

\section{First Order Reliability Method}

This method is used to calculate the failure probability of the evaluated integral in the standard Gaussian space using equation (12):

$$
P_{F}=\int I_{F}(\xi) f(\xi) d \xi=\int_{F} f(\xi) d \xi
$$

Assuming a failure region ' $F$ ' which is bounded by the thresholds values and $\xi$ represents the points located in this region. Thus, a standard Gaussian PDF can be assumed to decay as the distance of छfrom the origin increases. Also, assuming that $\xi^{\wedge *}$ has the highest PDF value among all other points, it can be stated that the surrounding points of $\xi^{\wedge *}$ have the major contribution to the failure probability. Therefore the probability of failure in this method is found by approximating the failure region ' $F$ ' and considering only the region with the highest PDF points which can be noted as 'F1'[12].

F1 can be evaluated using the following equation (13):

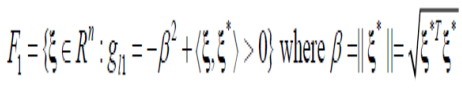

Where $\beta$ is the reliability index. Finally the failure probability is cal- 
culated by subsisting F with F1 in the following integral

$$
P(F)=\int_{F} f(E) d E \approx \int_{F_{1}} f(\xi) d E
$$

\section{Bayesian Updating for Model Parameters}

Bayesian updating in this research was used to update the model parameters. This was done by considering the predictions from equation (2) as our prior and the results from our inspection as our likelihood function. Then the posterior probability was found using the following equation (15):

$$
p\left(\boldsymbol{\theta} \mid \mathbf{D}_{M}, \mathcal{M}\right)=\frac{p\left(\hat{\mathbf{Y}}_{M} \mid \hat{\mathbf{U}}_{M}, \boldsymbol{\theta}, \mathcal{M}\right) p(\boldsymbol{\theta} \mid \mathcal{M})}{P\left(\hat{\mathbf{Y}}_{M} \mid \hat{\mathbf{U}}_{M}, \mathcal{M}\right)}
$$

Where $\theta$ represents the model parameters, $M$ is the model in equation (2), DM is the data provided from the Non-destructive inspection methods and YM and UM are the measurements for our output and input respectively. Considering the dependence of the output on $\theta$ and the independence of the inputs. The optimal values of the model parameters were evaluated using two different approaches Maximum Likelihood Estimate (MLE) and Maximuma Posterior (MAP). Maximum Likelihood Estimate (MLE) is defined by the following equation (16):

$$
\theta_{M L E}=\underset{\theta \in \Theta}{\arg \max } p\left(\hat{\mathbf{Y}}_{M} \mid \hat{\mathbf{U}}_{M}, \theta, \mathcal{M}\right)
$$

Where the updated parameters are assumed to maximize the likelihood of getting close values to the inspection or observation results DM in future predictions. Maximum a Posterior is defined by the following equation(17):

$$
\begin{aligned}
\boldsymbol{\theta}_{M A P} & =\underset{\theta \in \Theta}{\arg \max } p\left(\boldsymbol{\theta} \mid \mathbf{D}_{M}, \mathcal{M}\right) \\
& =\underset{\theta \in \Theta}{\arg \max } p\left(\hat{\mathbf{Y}}_{M} \mid \hat{\mathbf{U}}_{M}, \boldsymbol{\theta}, \mathcal{M}\right) p(\boldsymbol{\theta} \mid \mathcal{M})
\end{aligned}
$$

The parameters are considered to be the most probable values

Table 1: Statistical parameters for deterioration model random variables.

\begin{tabular}{|c|c|c|c|}
\hline Parameter & Mean & CV & Distribution \\
\hline $\mathrm{C}_{\mathrm{o}}\left(\mathrm{kg} / \mathrm{m}^{3}\right)$ & 3.5 & 0.5 & Log-normal \\
\hline $\mathrm{x}($ cover$)$ & $6 \mathrm{~cm}$ & 0.1 & Normal \\
\hline $\mathrm{E}_{\text {mod }}$ & 1 & 0.2 & Log-normal \\
\hline $\mathrm{D}_{(\text {Nominal) }}$ & $5 \times 10^{-5}$ & & Normal \\
\hline error(D) & 1 & 0.2 & Uniform \\
\hline $\mathrm{C}_{\mathrm{th}}\left(\mathrm{kg} / \mathrm{m}^{3}\right)$ & 0.9 & 0.19 & $(0.6-1.2)$ \\
\hline
\end{tabular}

for the model parameters based on the measured inspection data and the prior information about the parameters. In this paper a scalar Gaussian prediction error was considered that has a zero mean and an assumed standard deviation $\left(\sigma_{\mathrm{e}}\right)$. This will help in indicating the difference between the results of prediction model ' $z$ ' and the actual output ' $y$ '[12]. The system output can be modelled as following:

$$
y=z+e
$$

Where ' $\mathrm{e}$ ' is the model error.

\section{Results and Discussion}

This section will discuss the results associated with the three analysis stages; predicting corrosion initiation time, evaluating the probability of failure and Bayesian updating.

\section{Stage 1 Prediction of corrosion initiation time}

In this stage the corrosion initiation time $\left(\mathrm{t}_{0}\right)$ was predicted by applying MCS, IS and Taylor approximation on equation (2). It should be noted that 1000 simulations were performed. In MCS the samples where first generated directly from the parameters distributions. This gave a $\left(\mathrm{t}_{0}\right)$ equal to 31.5 years with $\mathrm{CV}$ equal to $1.6 \%$. In the second attempt the samples were generated in the standard to $1.5 \%$ which is relatively close to the first approach. In IS the optimum parameter values where obtained, then an importance sampling density was proposed for each component in the model. The standard deviation was the same but the mean value was updated with the optimum values. Table (2) summarizes the optimum value for the model parameters (Table 2).

IS gave an (to) equal to 31.33 years with a CV equal to $0.2 \%$. Figure (1) compares between MCS and IS in the standard Gaussian space. IS converged to the mean value after less number of iterations and with lower uncertainty than MCS (Figure 1,2).

In Taylor approximation the Hessian value was obtained using optimization and plugged in equation (10) to calculate the value of $\left(t_{0}\right)$. Table (3) summarizes the results obtained from the three different methods. Although, MCS converged after many number of iterations, it was the simplest stochastic method to perform (Table $3)$. Gaussian space this gave $\left(\mathrm{t}_{\mathrm{o}}\right)$ equal to 31.17 years with a CV equal

Coefficient of variation $(\mathrm{CV})$ 
Table 2: Optimized Model Parameters.

\begin{tabular}{|c|c|}
\hline Parameters & Optimum value \\
\hline $\mathrm{C}_{\mathrm{o}}$ & $3.38 \mathrm{~kg} / \mathrm{m}^{3}$ \\
\hline $\mathrm{X}$ & $6.1 \mathrm{~cm}$ \\
\hline $\mathrm{E}_{\text {mod }}$ & 0.93 \\
\hline $\operatorname{error}(\mathrm{D})$ & 0.96 \\
\hline $\mathrm{C}_{\mathrm{th}}$ & $1.019 \mathrm{~kg} / \mathrm{m}^{3}$ \\
\hline
\end{tabular}

Table 3: Stage 1Results from Stochastic Methods.

\begin{tabular}{|c|c|c|c|}
\hline Values & MCS & IS & Taylor \\
\hline $\mathrm{t}_{\mathrm{o}}(\mathrm{mean})$ & 31.17 & 31.336 & 31.2 \\
\hline $\mathrm{CV}$ & $1.50 \%$ & $0.20 \%$ & - \\
\hline
\end{tabular}

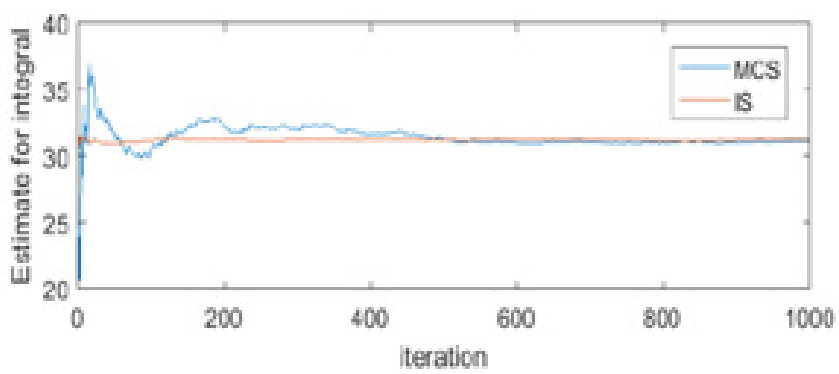

Figure 1: MCS and IS integral values vs iterations.

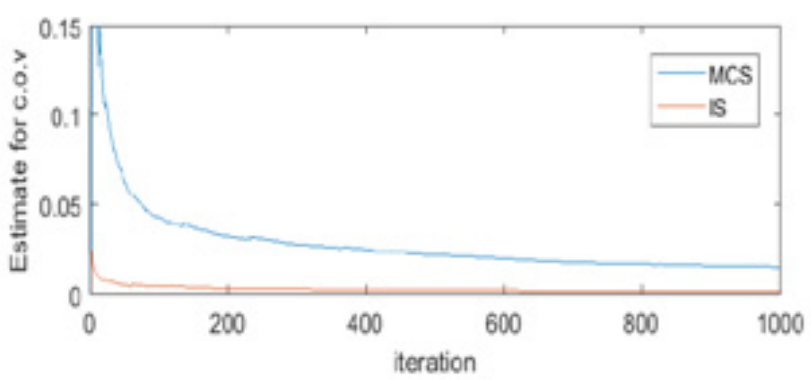

Figure 2: MCS and IS CV values vs iterations.

\section{Stage 2 Evaluating the probability of failure}

The critical chloride threshold $\left(\mathrm{C}_{\mathrm{th}}\right)$ value was first set to a deterministic value which is $0.9 \mathrm{~kg} / \mathrm{m}^{3}$ after that value is reached its assumed that corrosion will initiate in the concrete substrate [4]. It should be noted that 2000 simulations were performed. The probability of failure (Pf) was calculated using MCS, IS and FORM. All three methods were done in a standard Gaussian space. Figure (3) shows how the (Pf) increases with time and the difference between the three methods (Figure 3).

It was noted that after 30 years which is close to the predict corrosion initiation time, FORM method will provide inaccurate Pf values unlike the other two methods. Table (4) summarizes the results of the stochastic methods at 20 years and 30 years and the corresponding CV (Table 4).

From the results it can be observed that IS provides better certainty than MCS with slightly higher values for the Pf. As for the
FORM method it provided the highest Pf percentage at 20 years and lowest value at 30 years. A second approach was done in order to calculate the probability of failure. At this time the critical chloride threshold $\left(\mathrm{C}_{\mathrm{th}}\right)$ value was assumed a normal random variable associated with a standard deviation 0.17 and mean 0.9.MCS and FORM were used to calculate the probability of failure at 30 years in this approach. Also, the probability of failure was calculated analytically using the $\mathrm{CDF}$ associated with the $\left(\mathrm{C}_{\mathrm{th}}\right)$. In the second approach the MCS provided a value of $52.9 \%$ and a CV value $2 \%$. It can be noted that probability of failure increased as we increased the uncertainty in the model description. The analytical approach provided a probability of failure equal to $53.3 \%$ with a $\mathrm{CV}$ equal to $1.5 \%$. Since the $\mathrm{CV}$ varies according to the square root of the number of simulations, the computational efficiency increases by a factor of 1.78 when using an analytical approach. In the second approach FORM method provided a probability of failure $47 \%$ which is relatively higher than the Pf obtained when there was no uncertainty in our 
threshold value. It can be stated that as the standard deviation or the uncertainty of our threshold value increases the Pf increases.

\section{Stage 3Bayesian updating for model parameters}

The purpose of this stage is to update model parameters using inspection data (found in code). It was assumed that 20 measured points recording the concrete cover and the chloride content were obtained from Non-destructive inspection methods (i.e. cover meter and chloride ion penetration).The inspection was assumed to be done at year 20. A lognormal error of mean $0.5 \mathrm{~kg} / \mathrm{m}^{3}$ and a CV of $20 \%$ was chosen. The value of the CV was chosen based on the fact that the model was used before in many research and literature and the amount of error expected from it is relatively low $[2,4,9]$. It was found that two main factors affected the updated parameters, which are the quality of the data (i.e. dispersion and number of measurements) obtained from inspection and the assumed error in the model. Also, in this analysis the parameter ' $\mathrm{E}_{\bmod }$ ' which was multiplied by the model in stage 1 and 2 was ignored in the Bayesian updating stage. This was done due to the fact that the error factor was already added to the model. Table (5) compares the updated model parameters using both the MLE and MAP with the nominal values which were assumed to be the mean values of the parameters (Table 5).

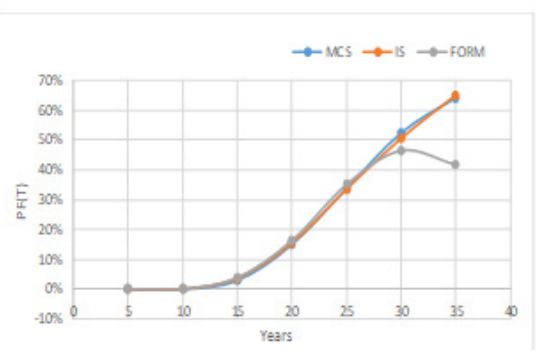

Figure 3: Probability of Failure Associated with Different Time Periods.

Figure 4: $\log (\operatorname{Pf}(b))$ vs $b$.

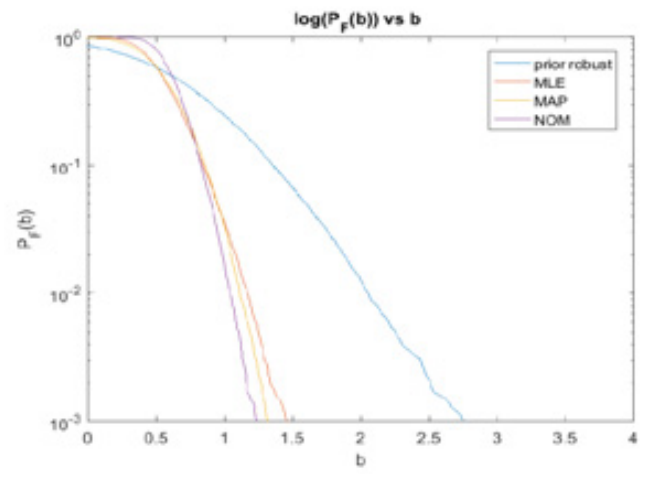

Bayesian updating was also used to predict the future behaviour of our model. The cover value was assumed to follow a normal distribution with a mean value of $6 \mathrm{~cm}$ and a $\mathrm{CV}$ equal to Table 4: Probability of failure at years 20 and 30

\begin{tabular}{|c|c|c|c|c|}
\hline Years & Values & MCS & IS & Form \\
\hline \multirow{2}{*}{20} & Pf $\%$ & $14.90 \%$ & $15.40 \%$ & $16.30 \%$ \\
\cline { 2 - 5 } & $\mathrm{CV}$ & $5 \%$ & $1.90 \%$ & -- \\
\cline { 2 - 5 } 30 & $\mathrm{Pf} \%$ & $50 \%$ & $51.50 \%$ & $46.40 \%$ \\
& $\mathrm{CV}$ & $2 \%$ & $1.30 \%$ & -- \\
\hline
\end{tabular}

Table 5: Model parameters nominal, MLE and MAP.

\begin{tabular}{|c|c|c|c|}
\hline Parameters & Nominal & MLE & MAP \\
\hline $\mathrm{C}_{\mathrm{o}}$ & $3.50 \mathrm{~kg} / \mathrm{m}^{3}$ & $5.72 \mathrm{~kg} / \mathrm{m}^{3}$ & $3.37 \mathrm{~kg} / \mathrm{m}^{3}$ \\
\hline error(D) & 1 & 0.649 & 0.926 \\
\hline $\boldsymbol{\sigma}_{\mathrm{e}}$ & -- & 0.074 & 0.178 \\
\hline $\mathrm{E}_{\bmod }$ & -- & -- & -- \\
\hline $\mathrm{x}$ (cover) & Measured on site & & \\
\hline
\end{tabular}




\section{Conclusion}

Prediction of the behavior of a Reinforced Concrete Bridge during its life span can help in making more optimum management decisions. Chloride induced corrosion is considered one of the main reasons behind concrete bridge deterioration. In this paper the corrosion initiation time and the probability of exceeding a critical chloride threshold value was estimated using stochastic methods. MCS, IS, Taylor approximation and FORM can assist in evaluating the prediction model. It was found that IS can provide future prediction with lower uncertainty and fewer iterations which makes it a more feasible method than MCS. It was observed that probability of failure increases with time. Also, assuming that the threshold value is a random variable associated with uncertainty might lead to an increase in the probability of failure. Bayesian updating helped in updating the model parameters using MLE and MAP. It was observed that both methods are affected by the quality of the inspection data provided and its number.

\section{Acknowledgement}

None.

\section{Conflict of Interest}

No conflict of interest.

\section{References}

1. Omar T, Nehdi ML, Zayed T (2017) Performance of NDT techniques in appraising condition of reinforced concrete bridge decks. Journal of Performance of Constructed Facilities 31(6): 4017104.

2. Chryssanthopoulos MK, Sterritt G (2002) Integration of deterioration modelling and reliability assessment for reinforced concrete bridge structures. In Proceedings of the Asranet International Colloquium.
3. Enright MP, Frangopol DM (1998) Probabilistic analysis of resistance degradation of reinforced concrete bridge beams under corrosion. Engineering Structures 20(11): 960-971.

4. Vu KAT, Stewart MG (2000) Structural reliability of concrete bridges including improved chloride-induced corrosion models. Structural Safety 22(4): 313-333.

5. Thomas O, Sobanjo J (2012) Comparison of Markov chain and semiMarkov models for crack deterioration on flexible pavements. Journal of Infrastructure Systems 19(2): 186-195.

6. Lounis Z (2003) Probabilistic modeling of chloride contamination and corrosion of concrete bridge structures. In Fourth International Symposium on Uncertainty Modeling and Analysis pp. 447-451.

7. Sarja A, Vesikari E (2014) Durability design of concrete structures. CRC Press, USA.

8. Stewart MG, Rosowsky DV (1998) Time-dependent reliability of deteriorating reinforced concrete bridge decks. Structural Safety 20(1): 91-109.

9. Rafiq MI, Chryssanthopoulos MK, Onoufriou T (2004) Performance updating of concrete bridges using proactive health monitoring methods. Reliability Engineering \& System Safety 86(3): 247-256.

10. Cheung MMS, So KKL (2015) Service Life Prediction and Management of Concrete Bridge Structures Due to Corrosion. Journal of Civil Engineering and Architecture 9: 1153-1166.

11. Duprat F (2007) Reliability of RC beams under chloride-ingress. Construction and Building Materials 21(8): 1605-1616.

12. Gaofeng J (2019) CIVE680B1: Stochastic Simulation in Engineering Applications.

13. Taylor BR, Qiao Y, Bowman MD, Labi S (2016) The Economic Impact of Implementing Nondestructive Testing of Reinforced Concrete Bridge Decks in Indiana.

14. Berg BA (2004) Markov Chain Monte Carlo simulations and their statistical analysis World Scientific Publishing. 